\title{
THE BINOMIAL EDGE IDEAL OF A PAIR OF GRAPHS
}

\author{
VIVIANA ENE, JÜRGEN HERZOG, \\ TAKAYUKI HIBI, AND AYESHA ASLOOB QURESHI
}

\begin{abstract}
We introduce a class of ideals generated by a set of 2-minors of an $(m \times n)$-matrix of indeterminates indexed by a pair of graphs. This class of ideals is a natural common generalization of binomial edge ideals and ideals generated by adjacent minors. We determine the minimal prime ideals of such ideals and give a lower bound for their degree of nilpotency. In some special cases we compute their Gröbner basis and characterize unmixedness and Cohen-Macaulayness.
\end{abstract}

\section{Introduction}

The study of ideals generated by minors of a generic matrix, mostly motivated by geometric questions, has a long tradition (see the fundamental papers [13] and [8] and the survey [2]). Classically, these are ideals generated by all minors of a given size. More recently, research has focused on ideals generated by arbitrary sets of minors of a generic matrix. Perhaps the first article in this direction is that of Andrade [1] from 1981 in which regular sequences of minors are considered. In the last years, due to techniques used in algebraic statistics, it proves necessary to study certain classes of binomial and determinantal ideals. This includes ideals generated by adjacent minors, as introduced by Diaconis, Eisenbud, and Sturmfels [4] and further studied in [9], [10], and [6], as well the binomial edge ideals, first considered in [7] and [11] and recently generalized in [12]. The algebraic properties of this class of ideals are widely open, although several partial results are known (see, e.g., [5]). From an algebraic point of view, we are interested in the following questions: What are the associated primes of these ideals and,

Received April 28, 2012. Accepted March 12, 2013.

First published online November 6, 2013.

2010 Mathematics Subject Classification. Primary 13P10, 13C13; Secondary 13C15, $13 \mathrm{P} 25$.

Ene's work was partially supported by Japan Society for the Promotion of Science Invitation Fellowship Programs for Research in Japan. 
in particular, their minimal primes? What is their Gröbner basis? When are these ideals reduced or prime? When are they Cohen-Macaulay or Gorenstein?

In this article, we introduce binomial edge ideals $J_{G_{1}, G_{2}}$ attached to a pair $\left(G_{1}, G_{2}\right)$ of finite graphs. This class of ideals generalizes the versions of binomial edge ideals, considered in [7], [11], and [12], but also includes ideals generated by adjacent minors which turn out to be the ideals attached to a pair of line graphs.

In Section 1 we study the Gröbner basis of these ideals. A general description of these Gröbner bases seems to be extremely difficult. However, in Theorem 1.3 we succeed in classifying those pairs of graphs for which $J_{G_{1}, G_{2}}$ has a quadratic Gröbner basis. Unlike the classical binomial edge ideals, the binomial edge ideals attached to a pair of graphs are never radical, unless $G_{1}$ or $G_{2}$ is complete (see Theorem 1.2).

In Theorem 2.7 we describe quite explicitly the minimal prime ideals of $J_{G_{1}, G_{2}}$. They are essentially determined by the so-called admissible sets of variables which are determined by data of the two graphs. The results obtained in Section 2 are applied in Section 3 to give a detailed description of all minimal prime ideals in the case that $G_{1}$ is a line graph of length 2 and $G_{2}$ is an arbitrary graph. The information on the minimal prime ideals is also used in Section 4, where the unmixed binomial edge ideals of pairs of graphs are characterized in Proposition 4.1. The condition for being unmixed is that one of the graphs is complete and the other graph satisfies certain numerical conditions related to its sets having the cut-point property. In the case that one graph is complete and the other one is a cycle, we fully classify in Proposition 4.2 the unmixed binomial edge ideals. Though the conditions guaranteeing that the binomial edge ideals of a pair of graphs are unmixed are already pretty restrictive, for them to be Cohen-Macaulay is even more restrictive. Under the assumption that $G_{1}$ is complete and that $G_{2}$ is closed in the sense of [7, Section 1] and $\left|V\left(G_{2}\right)\right| \geq\left|V\left(G_{1}\right)\right| \geq 3$, the unmixedness of $J_{G_{1}, G_{2}}$ is characterized and the depth of $S / J_{G_{1}, G_{2}}$ is computed (see Theorem 4.4). It follows that, under the assumptions of the theorem, $J_{G_{1}, G_{2}}$ is Cohen-Macaulay only if both graphs are complete.

For an ideal $I$ with radical $\sqrt{I}$, the least number $k$ with the property that $(\sqrt{I})^{k} \subset I$ is called the index of nilpotency of $I$ and is denoted nilpot $(I)$. It is clear that nilpot $(I)=1$ if and only if $I$ is a radical ideal. Thus, as noticed before, $\operatorname{nilpot}\left(J_{G_{1}, G_{2}}\right)=1$ if and only if $G_{1}$ or $G_{2}$ is complete. In the last section of this paper we give in Theorem 5.1 a lower bound for the index of 
nilpotency of $J_{G_{1}, G_{2}}$ in terms of data of graphs $G_{1}$ and $G_{2}$. Applying this result to an $(m \times n)$-matrix of adjacent minors, one obtains that this lower bound is approximately $m n / 16$.

It would be interesting to investigate how the construction we develop in this paper could be used to study minors of matrices that are not necessarily generic, for instance, scrolls.

\section{$\S 1$. Binomial edge ideals of pairs of graphs and their Gröbner basis}

Let $G_{1}$ be a graph on the vertex set $[m]$, and let $G_{2}$ be a graph on the vertex set $[n]$. We fix a field $K$; let $X=\left(x_{i j}\right)$ be an $(m \times n)$-matrix of indeterminates, and denote by $K[X]$ the polynomial ring in the variables $x_{i j}, i=1, \ldots, m$ and $j=1, \ldots, n$.

Let $e=\{i, j\}$ for some $1 \leq i<j \leq m$, and let $f=\{k, l\}$ for some $1 \leq k<$ $l \leq n$. To the pair $(e, f)$ we assign the following 2-minor of $X$ :

$$
p_{e, f}=[i, j \mid k, l]=x_{i k} x_{j l}-x_{i l} x_{j k} .
$$

The ideal

$$
J_{G_{1}, G_{2}}=\left(p_{e, f}: e \in E\left(G_{1}\right), f \in E\left(G_{2}\right)\right)
$$

is called the binomial edge ideal of the pair $\left(G_{1}, G_{2}\right)$.

EXAMPLES 1.1.

(a) If $G_{1}$ and $G_{2}$ are complete graphs, then $J_{G_{1}, G_{2}}=I_{2}(X)$, the ideal of all 2-minors of $X$.

(b) If $G_{1}$ is the graph consisting of exactly one edge, then $J_{G_{1}, G_{2}}$ is the binomial edge ideal $J_{G_{2}}$ introduced in [7].

(c) If $G_{1}$ is a complete graph, then $J_{G_{1}, G_{2}}$ is the generalized binomial edge ideal attached to $G_{2}$, as considered in [12].

(d) If $G_{1}$ and $G_{2}$ are line graphs, then $J_{G_{1}, G_{2}}$ is the ideal of adjacent 2-minors of the matrix $X$, studied in [4], [9], and [10].

THEOREM 1.2. Let $J_{G_{1}, G_{2}}$ be the binomial edge ideal of the pair of graphs $\left(G_{1}, G_{2}\right)$. Then the following conditions are equivalent:

(a) $J_{G_{1}, G_{2}}$ is a radical ideal, that is, $J_{G_{1}, G_{2}}=\sqrt{J_{G_{1}, G_{2}}}$;

(b) $J_{G_{1}, G_{2}}$ has a square-free Gröbner basis with respect to the lexicographic order induced by

$$
x_{11}>x_{12}>\cdots>x_{1 n}>x_{21}>x_{22}>\cdots>x_{m n} ;
$$

(c) either $G_{1}$ or $G_{2}$ is a complete graph. 
Proof. The implication (c) $\Rightarrow(\mathrm{b})$ is shown in [12, Theorem 18], and (b) $\Rightarrow$ (a) is a general fact (see, e.g., [7, proof of Corollary 2.2]). Thus, it remains to be shown that (a) implies (c). Suppose that neither $G_{1}$ nor $G_{2}$ is a complete graph. Then there exist subsets $T_{1} \subset[m]$ and $T_{2} \subset[n]$ such that the restrictions $L_{1}=\left(G_{1}\right)_{T_{1}}$ and $L_{2}=\left(G_{2}\right)_{T_{2}}$ are line graphs, each of them with two edges, say, $E\left(L_{1}\right)=\{\{i, j\},\{j, k\}\}$ and $E\left(L_{2}\right)=\{\{r, s\},\{s, t\}\}$. Then the element

$$
f_{L_{1}, L_{2}}=x_{i t} x_{j r} x_{k s}-x_{i r} x_{j s} x_{k t}
$$

does not belong to the ideal

$$
I=\left(p_{e, f}: e \in E\left(L_{1}\right), f \in E\left(L_{2}\right)\right)
$$

and hence $f_{L_{1}, L_{2}} \notin J_{G_{1}, G_{2}}$ because $I$ is obtained from $J_{G_{1}, G_{2}}$ by substituting by 0 all the variables which do not appear among the generators of $I$. On the other hand, $f_{L_{1}, L_{2}}^{2} \in I$, and hence $f_{L_{1}, L_{2}}^{2} \in J_{G_{1}, G_{2}}$. This shows that $J_{G_{1}, G_{2}}$ is not a radical ideal.

In [7] the concept of a closed graph is introduced. Recall that a graph $G$ on the vertex set $[n]$ is called closed if there exists a labeling of its vertices such that for all edges $\{i, j\}$ and $\{k, l\}$ of $G$ with $i<j$ and $k<l$, one has $\{j, l\} \in E(G)$ if $i=k$, and $\{i, k\} \in E(G)$ if $j=l$. Closed graphs are exactly those for which the classical associated binomial edge ideals have a quadratic Gröbner basis (see [7] and [3]).

The next result shows that only in exceptional cases do the binomial generators of $J_{G_{1}, G_{2}}$ form a Gröbner basis of $J_{G_{1}, G_{2}}$.

THEOREM 1.3. Let $J_{G_{1}, G_{2}}$ be the binomial edge ideal of the pair of graphs $\left(G_{1}, G_{2}\right)$. Then the following conditions are equivalent:

(a) $J_{G_{1}, G_{2}}$ has a quadratic Gröbner basis with respect to the monomial order introduced in Theorem 1.2,

(b) $G_{1}$ is complete and $G_{2}$ is closed, or vice versa.

Proof. The proofs consists of two parts.

$(\mathrm{a}) \Rightarrow(\mathrm{b})$ : Since the quadratic Gröbner basis of $J_{G_{1}, G_{2}}$ consists of binomials with square-free terms, it follows that $J_{G_{1}, G_{2}}$ is a radical ideal. Therefore, by Theorem 1.2, one of the graphs must be complete. Let us assume that $G_{1}$ is complete and show that $G_{2}$ is closed. Let $\{i, j\}$ be an edge of $G_{1}$, and let $\{k, l\},\{k, q\}$ be two edges of $G_{2}$ with $k<l$ and $k<q$. Then the 
$S$-polynomial $S(f, g)$ for $f=x_{i k} x_{j l}-x_{j k} x_{i l}$ and $g=x_{i k} x_{j q}-x_{i q} x_{j k}$ has the initial monomial $x_{j q} x_{j k} x_{i l}$, and since $J_{G_{1}, G_{2}}$ has quadratic Gröbner basis, we must have the edge $\{l, q\}$ in $G_{2}$.

(b) $\Rightarrow$ (a): Let $p_{e, f}, p_{e^{\prime}, f^{\prime}} \in J_{G_{1}, G_{2}}$. We show that $S\left(p_{e, f}, p_{e^{\prime}, f^{\prime}}\right)$ reduces to zero. If the initial terms of $p_{e, f}, p_{e^{\prime}, f^{\prime}}$ are coprime, then there is nothing to prove. Let $e=\{i, j\}$, let $f=\{k, l\}$, let $e^{\prime}=\left\{i^{\prime}, j^{\prime}\right\}$, and let $f^{\prime}=\left\{k^{\prime}, l^{\prime}\right\}$, with $i<j, k<l, i^{\prime}<j^{\prime}, k^{\prime}<l^{\prime}$. The initial terms of $p_{e, f}, p_{e^{\prime}, f^{\prime}}$ have a common factor if and only if (1) $i=i^{\prime}$ and $k=k^{\prime}$, (2) $j=j^{\prime}$ and $l=l^{\prime}$, or (3) $j=i^{\prime}$ and $l=k^{\prime}$. It is straightforward to verify in all three cases that $S\left(p_{e, f}, p_{e^{\prime}, f^{\prime}}\right)$ reduces to zero. For example, in case (1), if $j<j^{\prime}$ and $l<l^{\prime}$, then completeness of $G_{1}$ gives $g=\left\{j, j^{\prime}\right\} \in E\left(G_{1}\right)$, and closedness of $G_{2}$ implies that $h=\left\{l, l^{\prime}\right\} \in E\left(G_{2}\right)$. Then $S\left(p_{e, f}, p_{e^{\prime}, f^{\prime}}\right)$ reduces to zero with respect to $p_{e^{\prime}, h}$ and $p_{g, f}$.

\section{§2. The minimal prime ideals}

Let $J_{G_{1}, G_{2}} \subset K[X]$ be the binomial edge ideal of the pair of graphs $\left(G_{1}, G_{2}\right)$. Our aim is to describe the minimal prime ideals of $J_{G_{1}, G_{2}}$. This will be done in several steps. Throughout this section we will assume that $G_{1}$ and $G_{2}$ are both connected. This hypothesis is not restrictive. Indeed, let $G_{1 i}, 1 \leq i \leq r$, be the connected components of $G_{1}$, and let $G_{2 j}, 1 \leq j \leq s$, be the connected components of $G_{2}$. Then $J_{G_{1}, G_{2}}=\sum_{i, j} J_{G_{1 i}, G_{2 j}}$, and the generators of $J_{G_{1 i}, G_{2 j}}$ are binomials involving disjoint sets of variables of $X$. If $P$ is a minimal prime ideal of $J_{G_{1}, G_{2}}$, then for any $i, j$ there exists a minimal prime $P_{i j}$ of $J_{G_{1 i}, G_{2 j}}$ contained in $P$. This implies that $J_{G_{1}, G_{2}} \subset$ $\sum_{i, j} P_{i j} \subset P$. But since $\sum_{i, j} P_{i j}$ is a prime ideal, we must have $P=\sum_{i, j} P_{i j}$.

Lemma 2.1. The ideal $I_{2}(X)$ of all 2-minors of $X$ is a minimal prime ideal of $J_{G_{1}, G_{2}}$, and if $P$ is a minimal prime ideal of $J_{G_{1}, G_{2}}$ containing no variable, then $P=I_{2}(X)$.

Proof. Let $x=\prod_{\substack{i=1, \ldots, m \\ j=1, \ldots, n}} x_{i j}$. We claim that $J_{G_{1}, G_{2}}: x^{\infty}=I_{2}(X)$. This will then imply the assertions of the lemma, because if $P$ is a minimal ideal of $J_{G_{1}, G_{2}}$ not containing a variable, then $J_{G_{1}, G_{2}} \subset I_{2}(X)=J_{G_{1}, G_{2}}: x^{\infty} \subset$ $P: x^{\infty}=P$, and hence $P$ is equal to $I_{2}(X)$.

In order to prove the claim, let $\delta=[i, j \mid k, l]$ be an arbitrary 2-minor of $X$. We will show that $\delta \in J_{G_{1}, G_{2}}: x^{\infty}$. Assuming this, we conclude that $I_{2}(X): x^{\infty}=J_{G_{1}, G_{2}}: x^{\infty}$. However, since $I_{2}(X)$ is a prime ideal, we then have $I_{2}(X): x^{\infty}=I_{2}(X)$, and the claim is proved. 
To see that $\delta \in J_{G_{1}, G_{2}}: x^{\infty}$, we observe that there is a path $P_{1}$ in $G_{1}$ from $i$ to $j$, that is, a sequence $i=i_{0}, i_{1}, \ldots, i_{r-1}, i_{r}=j$ such that $\left\{i_{s}, i_{s+1}\right\} \in E\left(G_{1}\right)$ for $s=0, \ldots, r-1$. The number $r$ is called the length of the path. Similarly, there exists a path $P_{2}: k=k_{0}, k_{1}, \ldots, k_{t-1}, k_{t}=l$ in $G_{2}$ from $k$ to $l$. We will show by induction on $r+t$ that $\delta \in J_{G_{1}, G_{2}}: x^{\infty}$. Notice that $r+t \geq 2$. If $r+t=2$, then $\delta \in J_{G_{1}, G_{2}}$, and the assertion is trivial. Suppose now that $r+t>2$. We may assume that $r>1$. By applying the induction hypothesis, we have that $\delta_{1}=\left[i, i_{r-1} \mid k, l\right]$ and $\delta_{2}=\left[i_{r-1}, j \mid k, l\right]$ belong to $J_{G_{1}, G_{2}}: x^{\infty}$. Since $x_{i_{r-1} k} \delta=x_{i k} \delta_{2}+x_{j k} \delta_{1}$, it follows that $\delta \in J_{G_{1}, G_{2}}: x^{\infty}$, as desired.

Corollary 2.2. $J_{G_{1}, G_{2}}$ is a prime ideal if and only if $G_{1}$ and $G_{2}$ are complete graphs.

Next we will study minimal prime ideals of $J_{G_{1}, G_{2}}$ which contain variables. In this context, the following definition turns out to be useful.

Definition 2.3. A subset $W \subset[m] \times[n]$ is called admissible with respect to $\left(G_{1}, G_{2}\right)$ if it satisfies the following property: whenever $(i, j) \in e \times f \cap W$ for some $e \in E\left(G_{1}\right)$ and some $f \in E\left(G_{2}\right)$, then $\{i\} \times f \subset W$ or $e \times\{j\} \subset W$.

Obviously, the empty set and the set $[m] \times[n]$ are admissible.

If $e=\{i, j\} \in E\left(G_{1}\right)$ and $f=\{k, l\} \in E\left(G_{2}\right)$, then the sets $\{i\} \times f,\{j\} \times f$, $e \times\{k\}$, and $e \times\{l\}$ are called the edges of $e \times f$. An admissible set $W$ with respect to $\left(G_{1}, G_{2}\right)$ is characterized by the property that if $W \cap(e \times f) \neq \emptyset$, then one of the edges of $e \times f$ is contained in $W$.

The significance of admissible sets for the study of the minimal prime ideals of $J_{G_{1}, G_{2}}$ becomes apparent by the next result.

Lemma 2.4. Let $P$ be a prime ideal containing $J_{G_{1}, G_{2}}$, and let $W=$ $\left\{(i, j): x_{i j} \in P\right\}$. Then $W$ is an admissible set.

Proof. Let $(i, j) \in W$. Then $x_{i j} \in P$. Assume that $(i, j) \in e \times f$, with $e=\{i, k\}$ and $f=\{j, l\}$. Then $x_{i j} x_{k l}-x_{i l} x_{k j} \in J_{G_{1}, G_{2}} \subset P$. This implies that $x_{i l} x_{k j} \in P$. Since $P$ is prime, we have either $x_{i l} \in P$ or $x_{k j} \in P$. If $x_{i l} \in P$, then $\{i\} \times f \subset W$. Otherwise, we have $x_{k j} \in P$, and then $e \times\{j\} \subset W$.

We call a subset $E \subset E\left(G_{1}\right) \times E\left(G_{2}\right)$ connected if for all $e \times f$ and $e^{\prime} \times f^{\prime}$ in $E$ there exist $e_{i} \times f_{i} \in E, i=1, \ldots, r$ such that $e \times f=e_{1} \times f_{1}, e^{\prime} \times f^{\prime}=$ $e_{r} \times f_{r}$, and $\left(e_{i} \times f_{i}\right) \cap\left(e_{i+1} \times f_{i+1}\right) \neq \emptyset$ for $i=1, \ldots, r-1$.

An arbitrary subset $E \subset E\left(G_{1}\right) \times E\left(G_{2}\right)$ can be uniquely written as a disjoint union of connected subsets of $E\left(G_{1}\right) \times E\left(G_{2}\right)$, called the connected components of $E$. 
LEMMA 2.5. Let $W \subset[m] \times[n]$ be an admissible set with respect to $\left(G_{1}, G_{2}\right)$. Then the connected components of

$$
W^{c}=\left\{e \times f: e \in E\left(G_{1}\right), f \in E\left(G_{2}\right), W \cap(e \times f)=\emptyset\right\}
$$

are of the form $E_{1} \times E_{2}$, where $E_{1} \subset E\left(G_{1}\right)$ and $E_{2} \subset E\left(G_{2}\right)$.

Proof. Let $e \times f$ and $e^{\prime} \times f^{\prime}$ belong to the same connected component $C$ of $W^{c}$. Then there exist $e_{i} \times f_{i} \in C, i=1, \ldots, r$, such that $e \times f=e_{1} \times f_{1}$, $e^{\prime} \times f^{\prime}=e_{r} \times f_{r}$, and $\left(e_{i} \times f_{i}\right) \cap\left(e_{i+1} \times f_{i+1}\right) \neq \emptyset$ for $i=1, \ldots, r-1$.

We have to show that $e \times f^{\prime} \in C$ and that $e^{\prime} \times f \in C$. We show this by induction on $r$. The assertion is trivial if $r=1$. Now let $r>1$, and assume that the assertion is already shown for $r-1$. Then, since $e_{2} \times f_{2}$ is connected in $C$ to $e_{r} \times f_{r}$ by a chain of length $r-1$, the induction hypothesis implies that $e_{2} \times f_{r}$ belongs to $C$. Similarly, since $e_{r-1} \times f_{r-1}$ is connected in $C$ to $e_{1} \times f_{1}$ by a chain of length $r-1$, we have $e_{1} \times f_{r-1}$ in $C$. Suppose that $e_{1} \times f_{r} \notin C$. Then $e_{1} \neq e_{2}$ and $f_{r-1} \neq f_{r}$, and moreover, $\left(e_{1} \times f_{r}\right) \cap$ $W \neq \emptyset$, say, $(i, j) \in\left(e_{1} \times f_{r}\right) \cap W$. Since $W$ is admissible, it follows that either $\{i\} \times f_{r} \in W$ or $e_{1} \times\{j\} \in W$. This implies that $\left(e_{2} \times f_{r}\right) \cap W \neq \emptyset$ or $e_{1} \times f_{r-1} \neq \emptyset$. It follows that $e_{2} \times f_{r} \notin C$ or $e_{1} \times f_{r-1} \notin C$, a contradiction. Hence, we conclude that $e \times f^{\prime}=e_{1} \times f_{r} \in C$. Similarly, one can show that $e^{\prime} \times f \in C$.

Let $W$ be an admissible subset of $G_{1} \times G_{2}$, and let $C_{1}, \ldots, C_{r}$ be the connected components of $W^{c}$ in the graph $G_{1} \times G_{2}$. The set of edges of $G_{1} \times G_{2}$ is defined to be the set $\left\{\{\{i, j\},\{k, l\}\}:\{i, j\} \in E\left(G_{1}\right),\{k, l\} \in\right.$ $\left.E\left(G_{2}\right)\right\}$. By Lemma 2.5, there exist subgraphs $G_{1 i} \subset G_{1}$ and $G_{2 i} \subset G_{2}$ such that $C_{i}=E\left(G_{1 i}\right) \times E\left(G_{2 i}\right)$. Since all $C_{i}$ are connected, it follows that the graphs $G_{1 i}$ and $G_{2 i}$ are connected and that

$$
W^{c}=\bigsqcup_{i} E\left(G_{1 i}\right) \times E\left(G_{2 i}\right),
$$

where $\bigsqcup$ denotes the disjoint union.

For a graph $G$, we define $\widehat{G}$ to be the complete graph on the vertex set $V(G)$. By using this notation, we define

$$
\widehat{W}^{c}=\bigsqcup_{i} E\left(\widehat{G}_{1 i}\right) \times E\left(\widehat{G}_{2 i}\right) .
$$

Obviously, the ideal

$$
P_{W}=\left(\left\{x_{i j}:(i, j) \in W\right\}, Q_{W}\right) \quad \text { with } Q_{W}=\left(p_{e, f}: e \times f \in \widehat{W}^{c}\right)
$$

is a prime ideal. 
Proposition 2.6. Let $V$ and $W$ be two admissible sets with respect to $\left(G_{1}, G_{2}\right)$. Then the following conditions are equivalent:

(a) $P_{V} \subsetneq P_{W}$

(b) $V \subsetneq W$, and for all $e \times f \subset \widehat{V}^{c} \backslash \widehat{W}^{c}$, an edge of $e \times f$ belongs to $W$.

Proof. We divide the proof into two parts.

(a) $\Rightarrow$ (b): Let $(i, j) \in V$. Then $x_{i j} \in P_{V} \subset P_{W}$. This implies that $(i, j) \in$ $W$. Therefore, $V \subset W$. The inclusion must be proper; otherwise, $P_{V}=P_{W}$. Assume that $e \times f \subset \widehat{V}^{c} \backslash \widehat{W}^{c}$. Then $p_{e, f} \in Q_{V} \backslash Q_{W}$. This implies that $p_{e, f} \in P_{W} \backslash Q_{W}$. Therefore, some corner of $e \times f$ belongs to $W$. Since $P_{W}$ is a prime ideal, an edge of $e \times f$ belongs to $W$.

(b) $\Rightarrow$ (a): The inclusion $V \subsetneq W$ implies that $\left\{x_{i j}:(i, j) \in V\right\} \subsetneq\left\{x_{i j}\right.$ : $(i, j) \in W\}$. If there exist $p_{e, f} \in Q_{V} \backslash Q_{W}$, then $e \times f \subset \widehat{V}^{c} \backslash \widehat{W}^{c}$. By our assumption, this implies that an edge of $e \times f$ belongs to $W$. Therefore, $p_{e, f} \in\left(x_{i j},\{i, j\} \in W\right)$. This shows that $P_{V} \subsetneq P_{W}$.

THEOREM 2.7. We have the following.

(a) Let $P$ be a minimal prime ideal of the binomial edge ideal $J_{G_{1}, G_{2}}$ of the pair $\left(G_{1}, G_{2}\right)$. Then there exists an admissible set $W \subset G_{1} \times G_{2}$ such that $P=P_{W}$.

(b) Let $W \subset G_{1} \times G_{2}$ be an admissible set. Then $P_{W}$ is a minimal prime ideal of $J_{G_{1}, G_{2}}$ if and only if for any admissible set $V \subset G_{1} \times G_{2}$ properly contained in $W$ there exists $e \times f \in \widehat{V}^{c} \backslash \widehat{W}^{c}$ such that no edge of $e \times f$ belongs to $W$.

Proof. The proof is divided as follows.

(a) Let $W=\left\{(i, j): x_{i j} \in P\right\}$. Then $\left(\left\{x_{i j}:(i, j) \in W\right\}, J_{G_{1}, G_{2}}\right) \subset P$, and $\left(\left\{x_{i j}:(i, j) \in W\right\}, J_{G_{1}, G_{2}}\right)=\left(\left\{x_{i j}:(i, j) \in W\right\}, Q\right)$, where $Q$ is generated by all minors $p_{e, f}$ such that $W$ does not contain an edge of $e \times f$. Hence, since $W$ is admissible, as is shown in Lemma 2.4, it follows that $Q=\left(\left\{p_{e, f}: e \times f \in\right.\right.$ $\left.\left.\widehat{W}^{c}\right\}\right)$. Now we apply Lemma 2.5 and conclude that $Q=\sum_{i=1}^{r} J_{G_{1 i}, G_{2 i}}$, where $C_{1}, \ldots, C_{r}$ are the connected components $\widehat{W}^{c}$ and $C_{i}=E\left(G_{1 i}\right) \times E\left(G_{2 i}\right)$, as described in Lemma 2.5 and the comments following it.

Thus, our discussion so far shows that $P$ is a minimal prime ideal of

$$
Q=\left(\left\{x_{i j}:(i, j) \in W\right\}, \sum_{i=1}^{r} J_{G_{1 i}, G_{2 i}}\right) .
$$

Since the summands $J_{G_{1 i}, G_{2 i}}$ in $Q$ are ideals in pairwise different sets of variables, it follows that $P=\left(\left\{x_{i j}:(i, j) \in W\right\}, \sum_{i=1}^{r} P_{i}\right)$, where each $P_{i}$ 
is a minimal prime ideal of $J_{G_{1 i}, G_{2 i}}$. None of the $P_{i}$ contains a variable. It follows therefore from Lemma 2.1 that $P_{i}=I_{2}\left(\left(x_{k l}\right)_{k \in V\left(G_{1 i}\right)}\right)$ for $i=1, \ldots, r$, as desired. $l \in V\left(G_{2 i}\right)$

(b) This follows from Proposition 2.6.

Among the minimal prime ideals of $J_{G_{1}, G_{2}}$ are those which are determined only by the data of $G_{1}$ (resp., data of $G_{2}$ ). To explain this, let $G$ be a finite simple graph on the vertex set $[n]$. A subset $S \subset G$ is said to have the cut-point property if each $i \in S$ is a cut point of the graph $G_{[n] \backslash S}$. In other words, $S$ has the cut-point property if, for all $i \in S$, the number of connected components of $G_{([n] \backslash S) \cup\{i\}}$ is smaller than that of $G_{[n] \backslash S}$.

Proposition 2.8. Let $S_{1} \subset V\left(G_{1}\right)=[m]$ and $S_{2} \subset V\left(G_{2}\right)=[n]$ be subsets with the cut-point property. Then $W_{1}=S_{1} \times[n]$ and $W_{2}=[m] \times S_{2}$ are admissible sets, and $P_{W_{1}}$ and $P_{W_{2}}$ are minimal prime ideals of $J_{G_{1}, G_{2}}$.

Proof. By symmetry, it is enough to show that $W_{1}$ is admissible and that $P_{W_{1}}$ is a minimal prime ideal. The set $W_{1}$ being admissible is obvious. Now let $V \subset W_{1}$ be an admissible set which is a proper subset of $W_{1}$. Then $V=T \times[n]$, where $T \subset S_{1}$ is a proper subset of $S$. Since $S$ has the cut-point property, it follows that $\left(G_{1}\right)_{[n] \backslash T}$ has fewer connected components than does $\left(G_{1}\right)_{[n] \backslash S}$. Let $G$ be a connected component of $\left(G_{1}\right)_{[n] \backslash T}$ which is not a connected component of $\left(G_{1}\right)_{[n] \backslash S}$. Then there exist two vertices $i, j \in V(G)$ which are not connected in $\left(G_{1}\right)_{[n] \backslash S}$. Therefore, for any $f \in E\left(G_{2}\right)$, the set $\{i, j\} \times f$ is contained in $\widehat{V}^{c} \backslash \widehat{W}^{c}$ and does not have any edge in $W$. Thus, it follows from Theorem $2.7(\mathrm{~b})$ that $P_{W_{1}}$ is a minimal prime ideal of $J_{G_{1}, G_{2}}$.

\section{$\S 3$. The case $3 \times n$}

In this section we aim to describe explicitly the minimal prime ideals of $J_{G_{1}, G_{2}}$ in the case that $\left|V\left(G_{1}\right)\right|=3$.

Let $G_{1}$ be a connected graph on vertex set [3], and let $G_{2}$ be a connected graph on vertex set $[n]$. The graph $G_{1}$ is either a path graph or a complete graph. In the case of a complete graph, the minimal prime ideals are known by $[12]$. Here we want to analyze the case when $G_{1}$ is a line graph with edges $\{1,2\}$ and $\{2,3\}$. 
Let $T$ be any subset of $[n]$, and let $C_{1}, \ldots, C_{r}$ be the connected components of $\left(G_{2}\right)_{[n] \backslash T}$. Furthermore, let $B$ be a subset of $[r]$. We set

$$
W_{T, B}=([3] \times T) \cup \bigcup_{j \in B}\left(\{2\} \times V\left(C_{j}\right)\right) .
$$

Note that $W_{T, B}$ is an admissible set with respect to $\left(G_{1}, G_{2}\right)$. We are going to prove that any admissible set $W$ for which $P_{W}$ is a minimal prime ideal of $J_{G_{1}, G_{2}}$, is of the form $W_{T, B}$, where $T$ and $B$ satisfy some extra conditions.

We first show the following.

Lemma 3.1. Let $P_{W}$ be a minimal prime ideal of $J_{G_{1}, G_{2}}$. Suppose that there exists some $(i, s) \in W$ with $i \in\{1,3\}$ and $s \in[n]$. Then $[3] \times s \subset W$.

Proof. Let

$$
W^{\prime}=\{(2, r):(2, r) \in W\} \cup \bigcup_{[3] \times\{r\} \subset W}[3] \times\{r\} .
$$

We first show that $W^{\prime}$ is an admissible set with respect to $\left(G_{1}, G_{2}\right)$. Let $(i, r) \in e \times f \cap W^{\prime}$ for some $e \in E\left(G_{1}\right)$ and for some $f \in E\left(G_{2}\right)$. If $[3] \times\{r\} \subset$ $W$, then [3] $\times\{r\} \subset W^{\prime}$; in particular, $e \times\{r\} \subset W^{\prime}$. Otherwise, we may assume that $i=2$ and that $[3] \times\{r\} \not \subset W$. Then $\{2\} \times f \in W$ because $W$ is admissible, and hence $\{2\} \times f \in W^{\prime}$. Therefore, $W^{\prime}$ is admissible.

Assume that $W^{\prime} \neq W$. We claim that in this case $P_{W^{\prime}}$ is properly contained in $P_{W}$, contradicting the assumption that $P_{W}$ is a minimal prime ideal. Indeed, $W^{\prime}$ is a proper subset of $W$. Let $e \times f \in \widehat{W}^{\prime c} \backslash \widehat{W}^{c}$. We may assume that $e=\{1,2\}$. Then $\{1\} \times f \subset W$ because $\{2\} \times f \not \subset W$ and $W$ is admissible.

In the following, we will have to refer to the following operations on graphs. Let $G$ be a graph and let $H$ be a subgraph of $G$. Then $G \backslash\{i\}$ denotes the subgraph of $G$ which is obtained by removing the vertex $i$ along with all the edges incident to $i$, and $H \cup\{i\}$ denotes the subgraph of $G$ which is obtained by adding to $H$ the vertex $i$ and all the edges of $G$ which connect $i$ with $H$.

Lemma 3.2. Let $P_{W}$ be a minimal prime ideal of $J_{G_{1}, G_{2}}$, and let $T=$ $\{a \in[n]:[3] \times\{a\} \in W\}$. Then $T$ has the cut-point property.

Proof. Assume that $T$ does not have the cut-point property. Then there exists an element $a \in T$ such that $\left(G_{2}\right)_{[n] \backslash T}$ has the same number of con- 
nected components as $\left(G_{2}\right)_{([n] / T) \cup\{a\}}$. This implies that there exists a unique connected component $D$ of $\left(G_{2}\right)_{[n] / T \cup\{a\}}$ which contains $a$ and such that $C=D \backslash\{a\}$ is connected.

We set $W^{\prime}=W \backslash([3] \times\{a\})$ if $W \cap([3] \times V(C))=\emptyset$; otherwise, we set $W^{\prime}=W \backslash\{(1, a),(3, a)\}$. By using Lemma 3.1, it follows that $W^{\prime}$ is of the form $W_{T, B}$ as described in (2). Therefore, $W^{\prime}$ is admissible.

We claim that $P_{W^{\prime}} \subsetneq P_{W}$. By using Proposition 2.6, it is enough to show

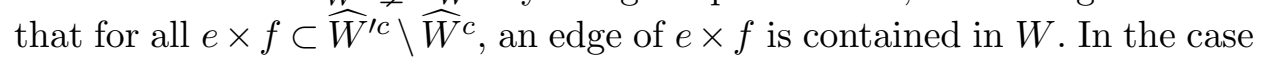
where $W^{\prime}=W \backslash([3] \times\{a\})$, any $e \times f \subset \widehat{W}^{\prime c} \backslash \widehat{W}^{c}$ has an edge in [3] $\times\{a\}$. In the case where $W^{\prime}=W \backslash\{(1, a),(3, a)\}$, we have $\widehat{W}^{\prime c}=\widehat{W}^{c}$. Therefore, our claim holds and we obtain a contradiction to the minimality of $P_{W}$.

Now we are ready to describe the minimal prime ideals of $J_{G_{1}, G_{2}}$.

THEOREM 3.3. Let $W$ be an admissible set with respect to $\left(G_{1}, G_{2}\right)$. Then the following conditions are equivalent:

(a) $P_{W}$ is a minimal prime ideal of $J_{G_{1}, G_{2}}$,

(b) $W=W_{T, B}$, where $T$ and $B$ satisfy the following conditions:

(i) $T$ has the cut-point property with respect to $G_{2}$,

(ii) let $C_{1}, \ldots, C_{r}$ be the connected components of $\left(G_{2}\right)_{[n] \backslash T}$; then

( $\alpha)\left|V\left(C_{j}\right)\right| \geq 2$ for $j \in B$,

$(\beta)$ for all $k, l \in B$ with $k \neq l,\left(C_{k} \cup C_{l}\right) \cup\{a\}$ is disconnected for all $a \in T$.

Proof. The proof is divided into two parts.

(a) $\Rightarrow(\mathrm{b})$ : We know from Lemmas 3.1 and 3.2 that $W=W_{T, B}$, where $T$ has the cut-point property with respect to $G_{2}$. Suppose that $\left|V\left(C_{j}\right)\right|=1$ for some $j \in B$; then $C_{j}=\{a\}$ for some $a \in V\left(G_{2}\right)$, and $W^{\prime}=W \backslash\{(2, a)\}$ is admissible with $P_{W^{\prime}} \subsetneq P_{W}$, a contradiction. This proves condition $(\alpha)$. Then suppose that there exists $a \in T$ such that $\left(C_{k} \cup C_{l}\right) \cup\{a\}$ is connected in $G_{2}$ for some $k, l \in B$ with $k \neq l$. Let $W^{\prime}=W \backslash\{(1, a),(3, a)\}$. Then $W^{\prime}$ is admissible and $P_{W^{\prime}} \subsetneq P_{W}$, a contradiction. This proves $(\beta)$.

(b) $\Rightarrow$ (a): Assume that $P_{W_{T, B}}$ is not a minimal prime ideal of $J_{G_{1}, G_{2}}$. Then there exists a minimal prime ideal $Q \subsetneq P_{W_{T, B}}$ of $J_{G_{1}, G_{2}}$. By the implication $(\mathrm{a}) \Rightarrow(\mathrm{b})$, which is already shown, it follows that $Q=P_{W_{T^{\prime}, B^{\prime}}}$, with $T^{\prime} \subset T$ and $B^{\prime} \subset B$. Suppose that $T^{\prime} \subsetneq T$. Since $T$ has the cut-point property, there exist two connected components $C_{k}, C_{l}$ of $\left(G_{2}\right)_{[n] \backslash T}$ and $a \in T \backslash T^{\prime}$ such that $\left(C_{k} \cup C_{l}\right) \cup\{a\}$ is connected. Let $i \in V\left(C_{k}\right)$, let $j \in V\left(C_{l}\right)$, and let $e \in E\left(G_{1}\right)$. Then $e \times\{i, j\}$ is contained in $\widehat{W}_{T^{\prime}, B^{\prime}}^{c} \backslash \widehat{W}_{T, B}^{c}$. It is clear 

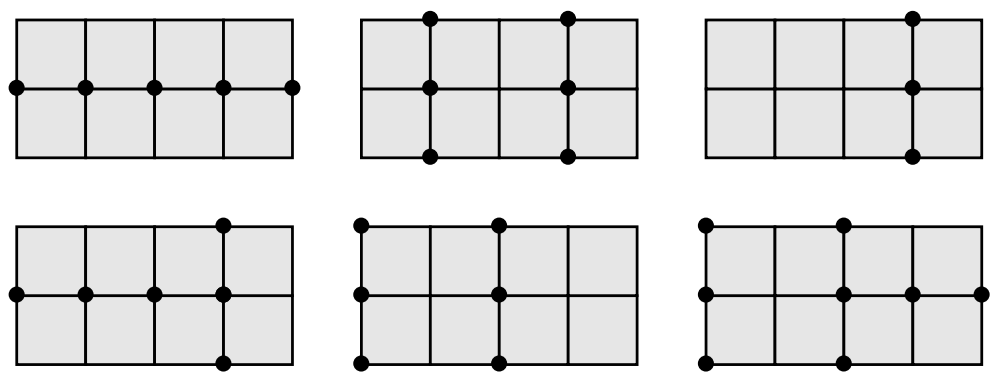

Figure 1: Admissible sets.

that the edges $e \times\{i\}, e \times\{j\}$, and $\{1\} \times\{i, j\}$, if $e=\{1,2\}$, respectively, and $\{3\} \times\{i, j\}$, if $e=\{2,3\}$, are not contained in $W_{T, B}$. But also the edge $\{2\} \times\{i, j\}$ is not contained in $W_{T, B}$ because of condition $(\beta)$. Therefore, it follows from Proposition 2.6 that $P_{W_{T^{\prime}, B^{\prime}}} \nsubseteq P_{W_{T, B}}$, a contradiction. Hence, we have $T^{\prime}=T$. Therefore, we must have $B^{\prime} \subsetneq B$. Then there exists $k \in B \backslash B^{\prime}$ such that $\left([3] \times V\left(C_{k}\right)\right) \cap W_{T, B^{\prime}}=\emptyset$. By condition $(\alpha)$, there exist $i, j \in V\left(C_{k}\right)$ with $i \neq j$. Therefore, $\{1,3\} \times\{i, j\}$ is contained in $\widehat{W}_{T, B^{\prime}}^{c} \backslash \widehat{W}_{T, B}^{c}$ and has no edge in $W_{T, B}$. It again gives a contradiction to our assumption that $P_{W_{T, B^{\prime}}} \subsetneq P_{W_{T, B}}$.

In [9, Theorem 3.1], Hoşten and Shapiro describe the minimal prime ideals of the ideal of adjacent 2 -minors of a $(3 \times n)$-matrix. In our language, these are the minimal prime ideals of $J_{G_{1}, G_{2}}$ where $G_{1}$ and $G_{2}$ are line graphs with $\left|V\left(G_{1}\right)\right|=3$ and $\left|V\left(G_{2}\right)\right|=n$. By using the fact that in this particular case the subsets $T=\left\{a_{1}, \ldots, a_{r}\right\}$ of $V\left(G_{2}\right)=[n]$ with the cut-point property are of the form $1<a_{1}, a_{r}<n$, and $a_{i}<a_{i+1}-1$ for $i=1, \ldots, r-1$, we obtained the result of Hoşten and Shapiro as a special case of Theorem 3.3.

In Figure 1, we display the admissible sets, marked by fat dots, attached with the minimal prime ideals of $J_{G_{1}, G_{2}}$, where $G_{1}$ is a line graph of length 2 and $G_{2}$ is a graph on vertex set [5] with edge set $\{\{1,2\},\{2,3\},\{3,4\},\{1,4\}$, $\{4,5\}\}$.

\section{§4. Unmixed binomial ideals of pairs of graphs}

In this section we classify all pairs of graphs $\left(G_{1}, G_{2}\right)$ such that $J_{G_{1}, G_{2}}$ is unmixed, and those for which $J_{G_{1}, G_{2}}$ is Cohen-Macaulay, under the additional assumption that the graphs are closed. 
For Cohen-Macaulayness, we may assume as well that $G_{1}$ and $G_{2}$ are connected since, with the same notation as at the beginning of Section 2,

$$
S / J_{G_{1}, G_{2}} \cong \bigoplus_{i j} S_{i j} / J_{G_{1 i}, G_{2 j}}
$$

where, for any $i, j$, the ring $S_{i j}$ is the polynomial ring over $K$ in the variables $X_{i j}=\left\{x_{k \ell}: k \in V\left(G_{1 i}\right), \ell \in V\left(G_{2 j}\right)\right\}$. Then, $J_{G_{1}, G_{2}}$ is Cohen-Macaulay if and only if $J_{G_{1 i}, G_{2 j}}$ is Cohen-Macaulay for any $i, j$.

Proposition 4.1. Let $n \geq m \geq 3$ be integers, and let $G_{1}$ and $G_{2}$ be connected simple graphs with $V\left(G_{1}\right)=[m]$ and $V\left(G_{2}\right)=[n]$. Then the binomial edge ideal $J_{G_{1}, G_{2}}$ is unmixed if and only if $G_{1}$ is complete and for all subsets $T \subset[n]$ with the cut-point property for $G_{2}$ one has

$$
(c(T)-1)(m-1)=|T| .
$$

Proof. Assume that $J_{G_{1}, G_{2}}$ is unmixed, and let us suppose that $G_{1}$ is not complete. Since $I_{2}(X)$ is one of the minimal primes of $J_{G_{1}, G_{2}}$ with height $(m-1)(n-1)$, all the other minimal prime ideals of $J_{G_{1}, G_{2}}$ must have the same height. By Proposition 2.8, any prime ideal $P_{W}$, where $W=S \times[n]$ and $\emptyset \neq S \subset[m]$ has the cut-point property for $G_{1}$, is a minimal prime of $J_{G_{1}, G_{2}}$. Let $G_{1}^{\prime}, \ldots, G_{c(S)}^{\prime}$ be the connected components of $\left(G_{1}\right)_{([m] \backslash S)}$, and let $g_{i}=\left|V\left(G_{i}^{\prime}\right)\right|$ for $i=1, \ldots, c(s)$. Then $\sum_{i=1}^{c(S)} g_{i}=m-|S|$ and

$$
\text { height } P_{W}=n|S|+\sum_{i=1}^{c(S)}\left(g_{i}-1\right)(n-1)=n|S|+(m-|S|-c(S))(n-1) \text {. }
$$

Hence, since $J_{G_{1}, G_{2}}$ is unmixed, we get $(c(S)-1)(n-1)=|S|$. Moreover, we have $|S| \geq n-1 \geq m-1$. But it is obvious that no $(m-1)$-subset of $[m]$ has the cut-point property for $G_{1}$; therefore, $G_{1}$ must be complete. By using arguments as in the first part of the proof for the graph $G_{2}$, one gets condition (3).

For the converse, we use [12, Theorem 23], which says that if $G_{1}$ is complete, then the minimal prime ideals of $J_{G_{1}, G_{2}}$ are exactly the prime ideals $P_{W}$ with $W=[m] \times T$, where $T \subset[n]$ is a set with the cut-point property for $G_{2}$. The numerical condition (3) shows that these prime ideals have all the same height; hence, $J$ is unmixed. 
Here Proposition 4.1 and Theorem 1.2 show, in particular, that an unmixed ideal associated with a pair of graphs is radical. It is very easy to see that the converse is not true. For instance, one may take $G_{1}$ the complete graph on [3] and $G_{2}$ the line graph with the edges $\{1,2\},\{2,3\}$. The ideal $J_{G_{1}, G_{2}}$ is radical, by Theorem 1.2 , and it is not unmixed, since its minimal prime ideals have different heights.

Proposition 4.1 shows also that $J_{G_{1}, G_{2}}$ is not unmixed for any connected graph $G_{2}$ which has a nonempty set $T$ with the cut-point property such that $m-1$ does not divide $|T|$. In particular, if $G_{2}$ is a tree, the ideal $J_{G_{1}, G_{2}}$ is not unmixed, since we may find subsets $T \subset[n]$ with the cut-point property of cardinality 1 . The next proposition addresses the unmixedness for the case when $G_{2}$ is a cycle.

Proposition 4.2. Let $n \geq m \geq 3$, let $G_{1}$ be the complete graph on $[m]$, and let $G_{2}$ be the cycle on the set $[n]$. Then $J_{G_{1}, G_{2}}$ is unmixed if and only if $m=n=3$, or $n=4, m=3$, or $n=5, m=3$.

Proof. By Proposition 4.1, $J_{G_{1}, G_{2}}$ is unmixed if and only if, for every subset $T \subset[n]$ which has the cut-point property for $G_{2}$, we have

$$
(c(T)-1)(m-1)=|T| .
$$

If $n \geq 6$, then there exist subsets $T$ of $[n]$ with the cut-point property such that $c(T)=|T|=3$. Hence, we get $2(m-1)=3$, which is impossible. Therefore, for unmixedness we must restrict to $n=3,4$, or 5 . If $m=n=3$, then the claims are obvious since $J_{G_{1}, G_{2}}$ is the ideal of all 2-minors of the matrix $X$.

Let $n=4$, and assume that $G_{2}$ has the edges $\{1,2\},\{2,3\},\{3,4\}$, and $\{4,1\}$. Then the sets with the cut-point property for $G_{2}$ are $\emptyset,\{1,3\}$, and $\{2,4\}$. By using (4) for a set $T$ with two elements, we get $m-1=2$; hence, $m=3$. In this case all the minimal prime ideals of $J_{G_{1}, G_{2}}$ have the same height equal to 6 .

Let $n=5$. In this case we see again that the nonempty subsets of [5] with the cut-point property for $G_{2}$ are of cardinality 2 , and, as in the case $n=4$, we obtain $m=3$.

REMARK 4.3. By using the computer, one easily sees that, in the hypotheses of the above proposition, $J_{G_{1}, G_{2}}$ is Cohen-Macaulay if and only if $m=$ $n=3$. 
Closed graphs form an interesting class of graphs $G_{2}$ for which one may discuss the unmixedness property. We recall that the collection of cliques of a graph $G$ forms a simplicial complex, called the clique complex of $G$. We denote it $\Delta(G)$. In [5, Theorem 2.2] it is shown that a graph $G$ on the vertex set $[n]$ is closed if and only if there exists a labeling of $G$ such that all the facets of $\Delta(G)$ are intervals $[a, b] \subset[n]$. Moreover, if one labels the facets $F_{1}, \ldots, F_{r}$ of $\Delta(G)$ such that $\min \left(F_{1}\right)<\min \left(F_{2}\right)<\cdots<\min \left(F_{r}\right)$, then $F_{1}, \ldots, F_{r}$ is a leaf order of $\Delta(G)$.

THEOREM 4.4. Let $n \geq m \geq 3$ be integers, let $G_{1}$ be the complete graph on $[m]$, and let $G_{2}$ be a connected closed graph on $[n]$. The following conditions are equivalent:

(i) $J_{G_{1}, G_{2}}$ is unmixed,

(ii) there exists a leaf order $F_{1}, \ldots, F_{r}$ of the facets of $\Delta\left(G_{2}\right)$ such that, for $1 \leq i \leq r, F_{i}=\left[a_{i}, b_{i}\right]$, where $a_{i}, b_{i}$ are positive integers with $a_{i}<a_{i+1}<$ $b_{i}<b_{i+1}$ and $b_{i}-a_{i+1}=m-2$ for $1 \leq i \leq r-1$.

Moreover, in the above conditions,

$$
\operatorname{depth}\left(S / J_{G_{1}, G_{2}}\right)=n-(r-2) m+2 r-3,
$$

where $r$ is the number of the facets of the clique complex $\Delta\left(G_{2}\right)$. Consequently, $S / J_{G_{1}, G_{2}}$ is Cohen-Macaulay if and only if $G_{2}$ is a complete graph.

Proof. By [5, Theorem 2.2], the clique complex $\Delta\left(G_{2}\right)$ has the facets $F_{1}, \ldots, F_{r}$, where each facet is an interval - that is, $F_{i}=\left[a_{i}, b_{i}\right]$, and $1=$ $a_{1}<a_{2}<\cdots<a_{r} \leq b_{r}=n$. Since $G_{2}$ is connected, it follows that $a_{i+1} \leq b_{i}$ for all $i$.

For $(\mathrm{i}) \Rightarrow$ (ii), we proceed by induction on $r$. Let $T=\left[a_{r}, b_{r-1}\right]$. Then $T$ has the cut-point property, and $c(T)=2$; thus, by (4), we get

$$
b_{r-1}-a_{r}+1=m-1
$$

Let $G_{2}^{\prime}$ be the graph whose clique complex $\Delta\left(G_{2}^{\prime}\right)$ has the facets $F_{1}, \ldots, F_{r-1}$, and let $P_{W^{\prime}}$ be a minimal prime of $J_{G_{1}, G_{2}^{\prime}}$, where $W^{\prime}=[m] \times T^{\prime}$, with $T^{\prime} \subset V\left(G_{2}^{\prime}\right)$ a set with the cut-point property for $G_{2}^{\prime}$. Then $b_{r-1} \notin T^{\prime}$; thus, $c_{G_{2}^{\prime}}\left(T^{\prime}\right)=c_{G_{2}}\left(T^{\prime}\right)$. It follows that $T^{\prime}$ has the cut-point property for $G_{2}$ as well. Therefore, $T^{\prime}$ satisfies condition (4), so we may apply induction.

For (ii) $\Rightarrow$ (i) and for the formula of the depth, we apply again induction on $r$. For $r=1$ there is nothing to prove since $J_{G_{1}, G_{2}}=I_{2}(X)$. In particular, $S / J_{G_{1}, G_{2}}$ is Cohen-Macaulay of depth $m+n-1$. 
Now let $r>1$, and let $G_{2}$ be a closed graph whose clique complex has $r$ facets, $F_{1}, \ldots, F_{r}$. For each subset $T$ of $[n]$ with the cut-point property for $G_{2}$, we denote by $P_{T}(J)$ the minimal prime ideal of $J=J_{G_{1}, G_{2}}$ which corresponds to the admissible set $W=[m] \times T$. Let $T_{0}=\left[a_{r}, b_{r-1}\right]$, and set

$$
J^{\prime}=\bigcap_{\substack{P_{T}(J) \in \operatorname{Min}(J) \\ T \not \supset T_{0}}} P_{T}(J), \quad J^{\prime \prime}=\bigcap_{\substack{P_{T}(J) \in \operatorname{Min}(J) \\ T \supset T_{0}}} P_{T}(J),
$$

where $\operatorname{Min}(J)$ is the set of the minimal prime ideals of $J$. Then $J=J^{\prime} \cap J^{\prime \prime}$; hence, in order to prove the unmixedness of $J$, we have to show that $J^{\prime}$ and $J^{\prime \prime}$ are unmixed of the same height equal to $(m-1)(n-1)$.

We note that $J^{\prime}=J_{G_{1}, G_{2}^{\prime}}$, where $G_{2}^{\prime}$ is obtained from $G_{2}$ by replacing the facets $F_{r-1}$ and $F_{r}$ of $\Delta\left(G_{2}\right)$ with the clique on the set $\left[a_{r-1}, n\right]$. Therefore, $G_{2}^{\prime}$ has $r-1$ cliques and $J^{\prime}$ is unmixed, by induction. In addition, again by induction, we get

$$
\operatorname{depth}\left(S / J^{\prime}\right)=n-(r-3) m+2 r-5
$$

On the other hand, $J^{\prime \prime}=\left(\left\{x_{i j}:(i, j) \in[m] \times T_{0}\right\}\right)+J_{G_{1}, G_{2}^{\prime \prime}}$, where $G_{2}^{\prime \prime}$ is the restriction of $G_{2}$ to the vertex set $[n] \backslash T_{0}$. It follows that $G_{2}^{\prime \prime}$ has two connected components; let us denote them $H_{1}$ and $H_{2}$, where $H_{1}$ is given by $r-1$ cliques on the vertex set $\left[a_{r}-1\right]$ and $H_{2}$ is the clique on the vertex set $\left[b_{r-1}+1, n\right]$. Therefore, by the inductive hypothesis, it follows that $J_{G_{1}, H_{1}}$ is unmixed of height $(m-1)\left(a_{r}-2\right)$. This implies that every minimal prime of $J^{\prime \prime}$ has height equal to $m\left|T_{0}\right|+(m-1)\left(a_{r}-2\right)+(m-1)\left(n-b_{r-1}-1\right)=$ $(m-1)(n-1)$; thus, $J^{\prime \prime}$ is also unmixed. This ends the proof of unmixedness of $J_{G_{1}, G_{2}}$.

In order to finish the proof of depth's formula, we use the following exact sequence:

$$
0 \rightarrow \frac{S}{J} \rightarrow \frac{S}{J^{\prime}} \oplus \frac{S}{J^{\prime \prime}} \rightarrow \frac{S}{J^{\prime}+J^{\prime \prime}} \rightarrow 0
$$

It is clear from the decomposition of $J^{\prime \prime}$ that

$$
\frac{S}{J^{\prime \prime}} \cong \frac{S_{1}}{J_{G_{1}, H_{1}}} \otimes_{K} \frac{S_{2}}{J_{G_{1}, H_{2}}}
$$

where $S_{1}$ is the polynomial ring in the variables $x_{i j},(i, j) \in[m] \times\left[a_{r}-1\right]$ and $S_{2}$ is the polynomial ring in the variables $x_{i j},(i, j) \in[m] \times\left[b_{r-1}+1, n\right]$. Since 
$J_{G_{1}, H_{1}}$ is unmixed and $H_{1}$ has $r-1$ cliques, by induction, it follows that $\operatorname{depth}\left(S_{1} / J_{G_{1}, H_{1}}\right)=a_{r}-1-(r-3) m+2 r-5=a_{r}-(r-3) m+2 r-6$. Since $H_{2}$ is a clique, we get $\operatorname{depth}\left(S_{2} / J_{G_{1}, H_{2}}\right)=n-b_{r-1}+m-1$. Consequently, by (6), we obtain

$$
\operatorname{depth}\left(S / J^{\prime \prime}\right)=n-(r-3) m+2 r-5 .
$$

Therefore,

$$
\operatorname{depth}\left(S / J^{\prime} \oplus S / J^{\prime \prime}\right)=n-(r-3) m+2 r-5 .
$$

Now we observe that $J^{\prime}+J^{\prime \prime}=J^{\prime}+\left(\left\{x_{i j}:(i, j) \in[m] \times T_{0}\right\}\right)+J_{G_{1}, G_{2}^{\prime \prime}}=$ $J^{\prime}+\left(\left\{x_{i j}:(i, j) \in[m] \times T_{0}\right\}\right)$ since $J_{G_{1}, G_{2}^{\prime \prime}}$ is obviously contained in $J^{\prime}$. But this shows that $S /\left(J^{\prime}+J^{\prime \prime}\right)$ is nothing else than $S / J_{G_{1}, H}$, where $H$ is the graph obtained from $G_{2}^{\prime}$ by replacing its last clique on the vertex set $\left[a_{r-1}, n\right]$ by the clique on the set $\left[a_{r-1}, n\right] \backslash T_{0}$. Therefore, $J_{G_{1}, H}$ is again unmixed and has $r-1$ cliques, so we may apply the inductive hypothesis. We then get

$$
\begin{aligned}
\operatorname{depth}\left(\frac{S}{J^{\prime}+J^{\prime \prime}}\right) & =\operatorname{depth}\left(\frac{S^{\prime}}{J_{G_{1}, H}}\right) \\
& =n-\left|T_{0}\right|-(r-3) m+2 r-5 \\
& =n-(r-2) m+2 r-4,
\end{aligned}
$$

where we denote by $S^{\prime}$ the polynomial ring in the variables $x_{i j}$ with $(i, j) \in$ $[m] \times\left([n] \backslash T_{0}\right)$. Finally, by applying the depth lemma in sequence (5), we get

$$
\operatorname{depth}(S / J)=\operatorname{depth}\left(S /\left(J^{\prime}+J^{\prime \prime}\right)\right)+1=n-(r-2) m+2 r-3 .
$$

The argument for the last claim in our theorem follows easily. If $G_{2}$ has $r$ cliques and if $J_{G_{1}, G_{2}}$ is Cohen-Macaulay, then the equality $m+n-1=$ $n-(r-2) m+2 r-3$ must hold. Then we get $(r-1) m=2 r-2$, which implies that $m=2$ or $r=1$. Hence, for $m \geq 3, G_{2}$ must be complete.

\section{§5. A lower bound for the nilpotency index of $J_{G_{1}, G_{2}}$}

Let $I$ be an ideal in a Noetherian ring. Then there exists an integer $k$ such that $(\sqrt{I})^{k} \subset I$, where $\sqrt{I}$ denotes the radical of $I$. We call the minimal number $k$ with this property the index of nilpotency of $I$ and denote it by 
$\operatorname{nilpot}(I)$. We have seen in Theorem 1.2 that nilpot $\left(J_{G_{1}, G_{2}}\right)=1$ if and only if either $G_{1}$ or $G_{2}$ is complete. In this section we want to give a lower bound for $\operatorname{nilpot}\left(J_{G_{1}, G_{2}}\right)$.

In the proof of the next result we will need the following concept. Let $I$ be an ideal in a polynomial ring $S$ over a field, and let $X$ be a set of variables of $S$. We say that $I$ is supported in $X$ if there exists a system of generators $f_{1}, \ldots, f_{l}$ of $I$ such that $X=\bigcup_{i=1}^{l} \operatorname{supp}\left(f_{i}\right)$, where for a polynomial $f$, $\operatorname{supp}(f)$ denotes the set of variables which appear in $f$. If $I$ is supported in $X$, we call $X$ a supporting set of $I$.

Theorem 5.1. Let $T_{1} \subset V\left(G_{1}\right)$, let $T_{2} \subset V\left(G_{2}\right)$, and let $C_{11}, \ldots, C_{1 r}$ and $C_{21}, \ldots, C_{2 s}$ be those connected components of $\left(G_{1}\right)_{T_{1}}$ and $\left(G_{2}\right)_{T_{2}}$, respectively, which contain as an induced subgraph a line graph of length at least 2. Then $\operatorname{nilpot}\left(J_{G_{1}, G_{2}}\right) \geq r s+1$.

Proof. In each $C_{i j}$ we choose a line graph $L_{i j}$ of length 2 which is an induced subgraph of $C_{i j}$, and let $f_{i j}=f_{L_{1 i} L_{2 j}}$, as defined in (1). Then, since $L_{1 i}$ and $L_{2 j}$ are also induced subgraphs of $G_{1}$ (resp., $G_{2}$ ), it follows that $f_{i j} \notin J_{G_{1}, G_{2}}$, but $f_{i j}^{2} \in J_{G_{1}, G_{2}}$, as shown in the proof of Theorem 1.2. Let $I$ be the ideal generated by the $f_{i j}$. Then $I \subset \sqrt{J_{G_{1}, G_{2}}}$. We claim that $f=$ $\prod_{\substack{i=1, \ldots, r \\ j=1, \ldots, s}} f_{i j}$ does not belong to $J_{G_{1}, G_{2}}$. This then implies that $I^{r s} \nsubseteq J_{G_{1}, G_{2}}$, and we obtain the desired inequality for the nilpotency index for $J_{G_{1}, G_{2}}$.

In order to prove the claim, let $L=\left(\left\{x_{k l}:(k, l) \in T_{1} \times T_{2}\right\}\right)$, and mark by the overline symbol reduction modulo $L$. Then $f=\bar{f}$ and

$$
\bar{J}_{G_{1}, G_{2}}=\sum_{\substack{i=1, \ldots, r \\ j=1, \ldots, s}} J_{C_{1 i}, C_{2 j}}+J_{0}
$$

where $J_{0}$ is the sum of the ideals of the form $J_{C, D}$ for the remaining connected components $C$ of $\left(G_{1}\right)_{T_{1}}$ and $D$ of $\left(G_{2}\right)_{T_{2}}$, which are different from the $C_{i j}$. Moreover, there exist supporting sets $X_{i j}$ for $J_{C_{1 i}, C_{2 j}}$ and $X_{0}$ for $J_{0}$ (resulting from the generating 2-minors of these ideals) such that $\operatorname{supp}\left(f_{i j}\right) \subset X_{i j}$ for all $i, j$, and such that all the supporting sets, including $X_{0}$, are pairwise disjoint.

Now suppose that $f \in J_{G_{1}, G_{2}}$. Then $f \in \bar{J}_{G_{1}, G_{2}}$ because $f=\bar{f}$. The next lemma, however, shows that $f \notin \bar{J}_{G_{1}, G_{2}}$, a contradiction. Thus, $f \notin J_{G_{1}, G_{2}}$. This proves the claim and the theorem. 
LEMMA 5.2. Let $I_{1}, \ldots, I_{r}$ be ideals in a polynomial ring $S$ with supporting sets $X_{1}, \ldots, X_{r}$, and let $f_{1}, \ldots, f_{r}$ be polynomials in $S$ such that $f_{j} \notin I_{j}$ for $j=1, \ldots, r$. Let $I=\sum_{i=1}^{r} I_{j}$, let $f=\prod_{i=1}^{r} f_{i}$, and suppose that

(i) $X_{i} \cap X_{j}=\emptyset$ for all $i \neq j$, and

(ii) $\operatorname{supp}\left(f_{i}\right) \subset X_{i}$ for all $i$.

Then $f \notin I$.

Proof. Choose any monomial order $<$ on $S$. It follows from (i) that in $_{<}(I)=\sum_{j=1}^{r}$ in $_{<}\left(I_{j}\right)$. Let $g_{j}$ be the remainder of $f_{j}$ with respect to a Gröbner basis of $I_{j}$. Since $f_{j}=g_{j}+h_{j}$ with $h_{j} \in I_{j}$, it follows that $f=$ $\prod_{i=1}^{r} g_{j}+h$, where $h \in I$. Hence, we see that $f \notin I$ if and only if $\prod_{i=1}^{r} g_{j} \notin I$. Thus, we may replace the $f_{j}$ by the $g_{j}$ and hence may assume from the very beginning that $\operatorname{in}_{<}\left(f_{j}\right) \notin$ in $_{<}\left(I_{j}\right)$.

Suppose that $f \in I$. Then $\operatorname{in}_{<}(f) \in \operatorname{in}_{<}(I)$, and therefore, $\prod_{i=1}^{r} \operatorname{in}_{<}\left(f_{i}\right) \in$ $\sum_{j=1}^{r}$ in $_{<}\left(I_{j}\right)$. This implies that for some $j$ there exists a monomial generator $u \in \operatorname{in}_{<}\left(I_{j}\right)$ such that $u$ divides $\prod_{i=1}^{r}$ in $_{<}\left(f_{i}\right)$. Since $\operatorname{supp}(u) \subset X_{j}$, it follows from (i) and (ii) that $u$ divides $\operatorname{in}_{<}\left(f_{j}\right)$. This is a contradiction, since $\operatorname{in}_{<}\left(f_{j}\right) \notin \operatorname{in}_{<}(I)$.

We give a concrete example of Theorem 5.1 in the form of the following corollary.

Corollary 5.3. Let $J$ be the ideal of adjacent minors of an $(m \times n)$ matrix, and let $k$ and $l$ be integers such that $m=4 k+p$ and $n=4 l+q$, with $0 \leq p, q<4$. Then

$$
\operatorname{nilpot}(J) \geq\left(k+\left\lfloor\frac{p}{3}\right\rfloor\right)\left(l+\left\lfloor\frac{q}{3}\right\rfloor\right)+1 \approx \frac{m n}{16} .
$$

In particular, the index of nilpotency of the binomial edge of a pair of graphs can be arbitrarily big.

Proof. We apply Theorem 5.1, in the case that $G_{1}$ is a line graph on $[m]$ and $G_{2}$ is a line graph on $[n]$. We choose $T_{1}=\{4 a: a \in[m], 4 a \leq m\}$ and $T_{2}=$ $\{4 b: b \in[n], 4 b \leq n\}$. Then Theorem 5.1 yields the desired conclusion.

The bound given in Corollary 5.3 is definitely not the best possible. Calculations by computer show that for $k=2,3,4$ the index of nilpotency of the ideal of adjacent 2-minors of a $(3 \times 3 k)$-matrix is at least $k+1$, whereas our Corollary 5.3 gives only $k$ as the lower bound for the index of nilpotency in these cases. 
Acknowledgments. We would like to thank the referees for a careful reading and for useful comments. Part of this article was written during the visit of the second author to Abdus Salam School of Mathematical Sciences, and he thanks that institute for its hospitality. The fourth author contributed to this paper during her postdoctoral fellowship at Abdus Salam School of Mathematical Sciences, for which she expresses her gratitude.

\section{REFERENCES}

[1] J. F. Andrade, Regular sequences of minors, Comm. Algebra 9 (1981), 765-781. MR 0609222. DOI 10.1080/00927878108822617.

[2] W. Bruns and A. Conca, "Gröbner bases and determinantal ideals" in Commutative Algebra, Singularities and Computer Algebra (Sinaia, 2002), NATO Sci. Ser. II Math. Phys. Chem. 115, Kluwer, Dordrecht, 2003, 9-66. MR 2030262.

[3] M. Crupi and G. Rinaldo, Binomial edge ideals with quadratic Gröbner bases, Electron. J. Combin. 18 (2011), paper 211. MR 2853068.

[4] P. Diaconis, D. Eisenbud, and B. Sturmfels, "Lattice walks and primary decomposition" in Mathematical Essays in Honor of Gian-Carlo Rota (Cambridge, Mass., 1996), Progr. Math. 161, Birkhäuser, Boston, 1998, 173-193. MR 1627343.

[5] V. Ene, J. Herzog, and T. Hibi, Cohen-Macaulay binomial edge ideals, Nagoya Math. J. 204 (2011), 57-68. MR 2863365.

[6] J. Herzog and T. Hibi, Ideals generated by adjacent 2-minors, J. Commut. Algebra 4 (2012), 525-549. MR 3053451. DOI 10.1216/JCA-2012-4-4-525.

[7] J. Herzog, T. Hibi, F. Hreinsdóttir, T. Kahle, and J. Rauh, Binomial edge ideals and conditional independence statements, Adv. in Appl. Math. 45 (2010), 317-333. MR 2669070. DOI 10.1016/j.aam.2010.01.003.

[8] M. Hochster and J. A. Eagon, Cohen-Macaulay rings, invariant theory, and the generic perfection of determinantal loci, Amer. J. Math. 93 (1971), 1020-1058. MR 0302643.

[9] S. Hoşten and J. Shapiro, "Primary decomposition of lattice basis ideals" in Symbolic Computation in Algebra, Analysis, and Geometry (Berkeley, 1998), J. Symbolic Comput. 29, Elsevier, Amsterdam, 2000, 625-639. MR 1769658. DOI 10.1006/jsco. 1999.0397.

[10] S. Hoşten and S. Sullivant, Ideals of adjacent minors, J. Algebra 277 (2004), 615-642. MR 2067622. DOI 10.1016/j.jalgebra.2004.01.027.

[11] M. Ohtani, Graphs and ideals generated by some 2-minors, Comm. Algebra 39 (2011), 905-917. MR 2782571. DOI 10.1080/00927870903527584.

[12] J. Rauh and N. Ay, Robustness and conditional independence ideals, preprint, arXiv:1110.1338 [math.AC].

[13] B. Sturmfels, Gröbner bases and Stanley decompositions of determinantal rings, Math. Z. 205 (1990), 137-144. MR 1069489. DOI 10.1007/BF02571229. 
Viviana Ene

Faculty of Mathematics and Computer Science

Ovidius University

900527 Constanta

Romania

vivian@univ-ovidius.ro

Jürgen Herzog

Fachbereich Mathematik

Universität Duisburg-Essen

Campus Essen

45117 Essen

Germany

juergen.herzog@uni-essen.de

Takayuki Hibi

Department of Pure and Applied Mathematics Graduate School of Information Science and Technology

Osaka University

Toyonaka

Osaka 560-0043

Japan

hibi@math.sci.osaka-u.ac.jp

Ayesha Asloob Qureshi

Abdus Salam School of Mathematical Sciences

GC University

New Muslim Town

Lahore 54600

Pakistan

ayesqi@gmail.com 